\title{
Bulk and Edge excitations in a $\nu=1$ quantum Hall ferromagnet
}

\author{
Rashmi Ray \\ Physics Department, City College of the City University of New York
}

New York, NY 10031

\begin{abstract}
In this article, we shall focus on the collective dynamics of the fermions in a $\nu=1$ quantum Hall droplet. Specifically, we propose to look at the quantum Hall ferromagnet. In this system, the electron spins are ordered in the ground state due to the exchange part of the Coulomb interaction and the Pauli exclusion principle. The low energy excitations are ferromagnetic magnons. To provide a means for describing these magnons, we shall discuss a method of introducing collective coordinates in the Hilbert space of many-fermion systems. These collective coordinates are bosonic in nature. They map a part of the fermionic Hilbert space into a bosonic Hilbert space. Using this technique, we shall interpret the magnons as bosonic collective excitations in the Hilbert space of the many-electron Hall system. By considering a Hall droplet of finite extent, we shall also obtain the effective Lagrangian governing the spin collective excitations at the edge of the sample.
\end{abstract}

${ }^{1}$ E-mail address: ray@aps.org. 


\section{Introduction}

Quantum Hall ferromagnets [1] have generated a considerable amount of interest in recent years. The fact that Hall states exhibit ferromagnetism is somewhat surprising. In conventional Hall systems, the electron spins are aligned by the external magnetic field. Thus in the absence of spontaneous alignment, the Hall states do not qualify as ferromagnetic states. However, particular samples, where the coupling of the electronic spins to the external magnetic field has been rendered negligible for a variety of reasons, do exhibit a spontaneous alignment of the spins. These are the Hall ferromagnets.

Traditionally, the distinction between the integer and the fractional Hall effects has been made based on the origin of the gap in the single particle spectrum. In the integer effects, the gap, (which is approximately equal to the cyclotron gap for $g \sim 2$ ) is produced by the Zeeman coupling of the electron spins to the external magnetic field. On the other hand, in the fractional effects, the gap is produced by the inter-electronic interactions.

There is, however, a scenario, where despite integer filling, the gap in the single particle spectrum is due to inter-electron interactions. For instance, in GaAs, the effective mass in the conduction band, which appears in the expression for the cyclotron gap, is much smaller than the actual mass of the electron, which appears in the expression for the Zeeman gap, through the Bohr magneton $\mu_{B}$. Thus the cyclotron gap increases effectively by a factor of $\sim 14$. Spin-orbit scattering reduces the effective $g$ factor by a factor of $\sim 5$. Hence the Zeeman energy is vanishingly small compared to the cyclotron energy (a factor of $\sim 70$ smaller).

Imagine that the $g$ factor in the sample can, starting from the value of 2 , be gradually brought down to zero. Initially, the $\nu=1$ state described a state with a uniform density of $\frac{B}{2 \pi}$ electrons per unit area, with each electron in the spin "up" state, say. With the reduction of the Zeeman gap, one would expect this state to be rendered unstable as the two spin orientations become degenerate. Experimentally, however, a gap is observed, indicating the $\nu=1$ state remains stable.

A rather heuristic argument may be adduced to explain this phenomenon. In the lowest Landau level, the kinetic energy of the electrons has a fixed value. Thus, to minimise the Coulomb energy, the spatial part of the many-electron wave function should be totally antisymmetric. This in turn will require, by the Pauli principle, the spin part of the wave function to be completely 
symmetric. Thus, the Coulomb interaction causes the spins to align and is instrumental in producing the observed gap. The state is genuinely ferromagnetic as the spins are spontaneously aligned. More importantly, the distinction between the integer and the fractional effects becomes somewhat blurred in this situation.

Since the ground state exhibits the spontaneous breaking of the original global spin $S U(2)$ symmetry (for $g=0$ ) down to a $U(1)$ symmetry, the excitation spectrum must contain gapless Goldstone bosons, namely, the ferromagnetic magnons.The effective Lagrangian governing the dynamics of the magnons has been obtained previously in a variety of ways [1],[7].

It is well known that the excitations located at the edges of finite Hall samples play a crucial role in the physics of these samples. The so-called edge states have been studied in considerable depth for the integer and the fractional effects [2],[3]. In contrast, despite some seminal work [4] on the edge excitatitons of ferromagnet Hall samples, a systematic derivation of the effective Lagrangian governing these excitations seems to be in order.

In this article, we propose to use a recently developed technique [5] for introducing bosonic collective variables as coordinates in many-fermion Hilbert spaces, in studying the bulk and the edge excitations of the $\nu=1$ Hall ferromagnet.

\section{Bosonic Effective Lagrangian for Collective Excitations of Fermions}

In this section, we shall outline a method [5] of introducing bosonic collective coordinates in the Hilbert space of many-fermion systems. These collective coordinates map a subspace of the full Hilbert space into a bosonic Hilbert space. We shall further obtain, starting from the second-quantised fermionic Lagrangian, an effective Lagrangian governing the bosonic collective coordinates.

Let us consider a system of $N$ fermions, free to reside at $K$ sites, with $K \geq N$. The number of orthogonal Fock states describing this system is obviously given by

$$
D_{\text {fermion }}=\frac{K !}{(K-N) ! N !}
$$

An arbitrary $N$-fermion determinantal wave function can be viewed as a tensor with $N$ antisym-

metric indices. The number of such independent tensors is $\frac{K !}{(K-N) ! N !}$. We immediately realise 
that $\frac{K !}{(K-N) ! N !}$ independent antisymmetric tensors form a basis of a completely antisymmetric irreducible representation of $S U(K)$. Thus, starting from any given state in the $N$-particle Fock space, we may arrive at any other by $S U(K)$ rotations.

Consider the fermionic second-quantised operators $\hat{c}_{\alpha}, \hat{c}_{\alpha}^{\dagger}$ satisfying $\left\{\hat{c}_{\alpha}, \hat{c}_{\beta}^{\dagger}=\delta_{\alpha \beta}\right.$ where $\alpha, \beta=$ $1,2, \cdots K$. Consider $U \in S U(K)$ and let $\hat{d} \equiv U \hat{c}$. Obviously, $\hat{d}$ satisfies the same canonical anticommutation relation as $\hat{c}$. Let us now construct a state

$$
|U\rangle \equiv \hat{d}_{1}^{\dagger} \hat{d}_{2}^{\dagger} \cdots \hat{d}_{N}^{\dagger}|0\rangle
$$

As argued above, by varying $U$, we may recover all the possible states in the Fock space. This enables us to keep the fermionic degrees of freedom "frozen" in a state with a fixed $U$ and to recover the dynamics through a variation of the bosonic degree of freedom $U$.

Let us define

$$
P \equiv \int d U|U\rangle\langle U|
$$

where $d U$ is the appropriate Haar measure for $S U(K)$. It can be shown that $P^{2}=P$. Thus $P$ is the projector onto the subspace of $N$-particle determinantal wave functions. In the sequel, we shall assume without proof that this is the appropriate subspace for the Hall ferromagnet.

The partition function of the system, projected onto this subspace, is readily obtained to be

$$
Z=\int \mathcal{D} U e^{i \int d t\left\langle U(t)\left|i \partial_{t}-H\right| U(t)\right\rangle}
$$

where $H$ is the second-quantised fermionic Hamiltonian.

Let us consider a fermionic Hamiltonian of the form

$$
H=\hat{c}_{\alpha}^{\dagger} h_{\alpha \beta} \hat{c}_{\beta}+\hat{c}_{\alpha_{1}}^{\dagger} \hat{c}_{\alpha_{2}}^{\dagger} V_{\alpha_{1} \alpha_{2}}^{\beta_{1} \beta_{2}} \hat{c}_{\beta_{2}} \hat{c}_{\beta_{1}}
$$

Now

$$
\left\langle U\left|\hat{d}_{\alpha}^{\dagger} \hat{d}_{\beta}\right| U\right\rangle \equiv \rho_{\alpha \beta}
$$

Obviously $\rho_{\alpha \beta}=\delta_{\alpha \beta}$ if $\alpha, \beta \leq N$ and is zero otherwise.

Then, from (1.4), the effective bosonic Lagrangian emerges as

$$
L_{e f f}=\operatorname{tr} \rho U^{\dagger} i \partial_{t} U-\operatorname{tr} \rho U^{\dagger} h U-\left(U \rho U^{\dagger}\right)_{\beta_{1} \alpha_{1}}\left(U \rho U^{\dagger}\right)_{\beta_{2} \alpha_{2}}\left[V_{\alpha_{1} \alpha_{2}}^{\beta_{1} \beta_{2}}-V_{\alpha_{1} \alpha_{2}}^{\beta_{2} \beta_{1}}\right]
$$


Lagrangians similar to this have been obtained in the literature [6].

\section{Planar Fermions in the Lowest Landau Level}

Before introducing the bosonic collective coordinates, let us briefly recapitulate [7] the basic physics of planar fermions subjected to a strong magnetic field orthogonal to the plane. We assume from the onset that the Zeeman coupling of the electron spins to this magnetic field is zero, due to the vanishingly small value of the effective g-factor.

The single particle (s.p.) Hamiltonian is the celebrated Landau Hamiltonian. If $\vec{A}$ is the gauge potential (we choose the symmetric gauge for convenience) giving rise to the external magnetic field, we have

$$
h_{0}=\frac{1}{2 m}(\vec{p}-\vec{A})^{2} \equiv \frac{1}{2 m} \vec{\Pi}^{2}
$$

where $\Pi^{x}=-i \partial_{x}-\frac{B}{2} y, \Pi^{y}=-i \partial_{y}+\frac{B}{2} x$ and defining $\pi \equiv \frac{1}{\sqrt{2 B}}\left(\Pi^{x}-i \Pi^{y}\right)$ and $\pi^{\dagger}$ as its complex conjugate, we have,

$$
\left[\pi, \pi^{\dagger}\right]=1
$$

The large degeneracy $\left(\frac{B}{2 \pi}\right.$ states per unit area) of the s.p. spectrum is expressed through the introduction of the guiding centre coordinates:

$$
\hat{X} \equiv \hat{x}-\frac{1}{B} \hat{\Pi}^{y}, \quad \hat{Y} \equiv \hat{y}+\frac{1}{B} \hat{\Pi}^{x}, \quad[\hat{X}, \hat{Y}]=\frac{i}{B}
$$

The holomorphic combination and its complex conjugate,

$$
\hat{a} \equiv \sqrt{\frac{B}{2}}(\hat{X}+i \hat{Y}), \quad \hat{a}^{\dagger} \equiv \sqrt{\frac{B}{2}}(\hat{X}-i \hat{Y})
$$

satisfy

$$
\left[\hat{\pi}, \hat{\pi}^{\dagger}\right]=1, \quad\left[\hat{a}, \hat{a}^{\dagger}\right]=1, \quad[\hat{a}, \hat{\pi}]=\left[\hat{a}, \hat{\pi}^{\dagger}\right]=\left[\hat{a}^{\dagger}, \hat{\pi}\right]=\left[\hat{a}^{\dagger}, \hat{\pi}^{\dagger}\right]=0
$$

These are related to the coordinate operators by

$$
\sqrt{\frac{B}{2}}(\hat{x}+i \hat{y}) \equiv \hat{z}=\hat{a}-i \hat{\pi}^{\dagger}, \quad \sqrt{\frac{B}{2}}(\hat{x}-i \hat{y}) \equiv \hat{\bar{z}}=\hat{a}^{\dagger}+i \hat{\pi}
$$

Of course $[\hat{z}, \hat{\bar{z}}]=0$. 
The eigenbasis of $h_{0}$ may be taken to be $|n, l\rangle$, where $n=0,1,2 \cdots \infty$ and the same is true for $l$. The index $n$ is called the Landau level index. $n=0$ corresponds to the lowest Landau level (L.L.L.). We have

$$
\hat{\pi}|n, l\rangle=\sqrt{n}|n-1, l\rangle, \hat{\pi}^{\dagger}|n, l\rangle=\sqrt{n+1}|n+1, l\rangle
$$

and

$$
\hat{a}|n, l\rangle=\sqrt{l}|n, l-1\rangle, \hat{a}^{\dagger}|n, l\rangle=\sqrt{l+1}|n, l+1\rangle .
$$

Thus,

$$
\hat{\pi}^{\dagger} \hat{\pi}|n, l\rangle=n|n, l\rangle, \hat{a}^{\dagger} \hat{a}|n, l\rangle=l|n, l\rangle .
$$

We can also define a coherent state basis for $\hat{a}[3]$. If

$$
|\xi\rangle \equiv e^{\xi \hat{a}^{\dagger}}|0\rangle
$$

we can easily check that $\hat{a}|\xi\rangle=\xi|\xi\rangle$. The inner product of two coherent states is given by $\langle\eta \mid \xi\rangle=e^{\bar{\eta} \xi}$ and the resolution of the identity is $\int d^{2} \xi e^{-|\xi|^{2}}|\xi\rangle\langle\xi|=I$, where $d^{2} \xi \equiv \frac{d \operatorname{Re} \xi d \operatorname{Im} \xi}{\pi}$. The coherent state basis is related to the $|l\rangle$ basis through $\langle l \mid \xi\rangle=\frac{\xi^{l}}{\sqrt{l}}$. The L.L.L. wave function is given by

$$
\langle\vec{x} \mid 0, l\rangle=\sqrt{\frac{B}{2 \pi}} \frac{1}{\sqrt{l}} e^{-\frac{1}{2}|z|^{2}} \bar{z}^{l}
$$

in the $|l\rangle$ basis and

$$
\langle\vec{x} \mid 0, \xi\rangle=\sqrt{\frac{B}{2 \pi}} e^{-\frac{1}{2}|z|^{2}+\bar{z} \xi}
$$

in the coherent state basis.

A smooth function of $\hat{z}, \hat{\bar{z}}$, may be expanded in the following manner:

$$
A(\hat{z}, \hat{\bar{z}})=A\left(\hat{a}-i \hat{\pi^{\dagger}}, \hat{a^{\dagger}}+i \hat{p}\right)=\left.\sum_{p, q} \frac{\left(-i^{p}\right) i^{q}}{p ! q !}\left(\hat{\pi^{\dagger}}\right)^{p}(\hat{\pi})^{q} \ddagger \partial_{z}^{p} \partial_{\bar{z}}^{q} A(z, \bar{z})\right|_{z=\hat{a}, \bar{z}=\hat{a^{\dagger}}} \ddagger
$$

Here, the symbol $\ddagger \cdots \ddagger$ indicates that since the $\hat{\pi}, \hat{\pi}^{\dagger}$ have been normal ordered, the $\hat{a}, \hat{a^{\dagger}}$ are automatically anti-normal ordered. Now when such a function is projected onto the L.L.L., only the term with $p=0, q=0$ in the sum over $p, q$ survives. Thus, in the L.L.L., the function $A(\hat{x}, \hat{y}) \rightarrow \ddagger A\left(\hat{a}, \hat{a^{\dagger}}\right) \ddagger$. It is instructive to express this anti-normal ordered operator in the coherent state basis. Let

$$
\ddagger A \ddagger \equiv \sum_{p, q} \frac{1}{p ! q !}(\hat{a})^{p}\left(\hat{a^{\dagger}}\right)^{q} A_{p q}
$$


We now insert the resolution of the identity in the coherent state basis between the $\hat{a}$ s and the $\hat{a}^{\dagger} \mathrm{s}$ in (2.14). We then obtain

$$
\ddagger A \ddagger=\int d^{2} \xi e^{-|\xi|^{2}}|\xi\rangle A(\xi, \bar{\xi})\langle\bar{\xi}|
$$

where $A(\xi, \bar{\xi}) \equiv \sum_{p, q} \frac{1}{p ! q !} A_{p q}(\xi)^{p}(\bar{\xi})^{q}$. Thus the product of two individually anti-normal ordered operators is given by

$$
\ddagger A \ddagger \ddagger B \ddagger=\int d^{2} \xi e^{-|\xi|^{2}}|\xi\rangle A(\xi, \bar{\xi}) * B(\xi, \bar{\xi})\langle\bar{\xi}|
$$

where the $*$ product is given by

$$
A(\xi, \bar{\xi}) * B(\xi, \bar{\xi}) \equiv \sum_{n=0}^{\infty} \frac{(-1)^{n}}{n !} \partial_{\bar{\xi}}^{n} A \partial_{\xi}^{n} B
$$

The star product is associative in that $(A * B) * C=A *(B * C)$. This concept of the star product should be very familiar to the aficionados of non-commutative field theories. In fact, the field theory of fermions in the L.L.L. is an instance of such a field theory, where the noncommutativity is restricted to the spatial coordinates.

Upto this point, we have considered only the s.p. Landau Hamiltonian. However, there are other contributions to the many particle Hamiltonian, which we shall now discuss.

The interaction between the electrons is the Coulomb interaction, whose contribution, as indicated in (1.7), splits naturally into a direct part and an exchange part. It is the exchange part which is instrumental in producing ferromagnetic behaviour in the Hall droplet.

Projected on to the L.L.L., the Coulomb term is written as:

$$
H_{c}=\frac{1}{2} \int d^{2} z_{1} d^{2} z_{2} e^{-\left|z_{1}\right|^{2}-\left|z_{2}\right|^{2}} \bar{\psi}_{\alpha}\left(z_{1}\right) \bar{\psi}_{\beta}\left(z_{2}\right) V\left(\sqrt{\frac{2}{B}}\left|z_{1}-z_{2}\right|\right) \psi_{\beta}\left(\overline{z_{2}}\right) \psi_{\alpha}\left(\overline{z_{1}}\right)
$$

where $V$ is the Coulomb interaction and $\psi_{\alpha}$ are the second quantised electron operators with spin $\alpha$, projected onto the L.L.L.. Let $\left.\left|\psi_{\alpha}\right\rangle\right\rangle$ be the abstract notation for the second quantised electron operator, with $\left.\left\langle\vec{x} \mid \psi_{\alpha}\right\rangle\right\rangle \equiv \psi_{\alpha}(\vec{x})$ being the corresponding field operator. Then projection to the L.L.L. entails:

$$
\left.\left.\left\langle\vec{x} \mid \psi_{\alpha}\right\rangle\right\rangle \equiv \psi_{\alpha}(\vec{x}) \rightarrow\left\langle\vec{x}\left|\sum_{l=0}^{\infty}\right| 0, l\right\rangle\left\langle 0, l \mid \psi_{\alpha}\right\rangle\right\rangle .
$$

We define $\left.c_{\alpha}(l) \equiv\langle 0, l \mid \psi\rangle\right\rangle$. This is the operator that destroys an electron with index $l$ and spin $\alpha$. Then upon using (2.11), we have

$$
\psi_{\alpha}(\vec{x}) \rightarrow \sqrt{\frac{B}{2 \pi}} \sum_{l=0}^{\infty} \frac{1}{\sqrt{l}} e^{-\frac{1}{2}|z|^{2}} \bar{z}^{l} c_{\alpha}(l) \equiv e^{-\frac{1}{2}|z|^{2}} \psi_{\alpha}(\bar{z}) .
$$


This is the $\psi(\bar{z})$ that has been used in $(2.18)$.

Apart from the Coulomb interaction, we require that the electrons be confined to a finite portion of the plane. This entails the introduction of a suitable confining potential [5].

Let us introduce a radially symmetric confining potential $v(r)$ that confines $N$ electrons in a droplet of radius $R$.

$$
v(\hat{z}, \hat{\bar{z}})=\gamma|\hat{z}|^{2}
$$

where $\gamma$ is the strength of the confining potential.

In view of the fact that the coordinate operators do not commute when projected to the L.L.L.. The confining potential is really the Hamiltonian of a one dimensional harmonic oscillator, with the coordinate operators acting as canonically conjugate variables. The eigenvalues of $v$ are therefore given by

$$
\epsilon_{n}=\gamma\left(n+\frac{1}{2}\right), n=0,1,2, \cdots \infty
$$

If we fill the available single particle states with $N$ particles to form a droplet with the lowest possible energy, the energy of this droplet would be

$$
E_{t o t} \equiv \sum_{n=0}^{N-1} \epsilon_{n}=\gamma \frac{N^{2}}{2}
$$

We know that the degeneracy in the L.L.L. is given by $\frac{B}{2 \pi}$ particles per unit area. If the radius of the droplet be $R$, we have the relation

$$
N=\left(\frac{B}{2 \pi}\right)\left(\pi R^{2}\right) \Rightarrow N=\frac{B R^{2}}{2}
$$

Now, if the magnetic field is held fixed and we increase the number of electrons, the droplet obviously increases in size. However, from (2.23), it is clear that $E_{t o t} \sim N^{2}$ as this is done. Thus, from (2.24), it seems that the energy increases quadratically with the size of the sample. However, for the energy to be a properly extensive quantity, we require that it should be directly proportional to the area (i.e. to $N$ ). The way to resolve this is to consider a confining potential whose strength is of order one (in $N$ ) in the bulk but is of order $N$ at the boundary. This form of the confining potential shall be seen to have a crucial significance in determining the leading order terms in the bosonic effective Lagrangian that we shall compute further on. 
The ground state of the many electron system is formed by filling up the s.p. states of the confining potential sequentially with electrons. Furthermore, as is well known, the ground state of the system is ferromagnetic for large $N$. Thus the electrons in the ground state all have say, spin "up". Let us now try to relate all this to the formulation of the collective theory given in section I. The available sites ( $K$ in number) correspond to the s.p. eigenstates of the confining potential (labelled by integers). Furthermore, each state has two values of the spin index associated with it. Thus, in line with what we have said in section I, the bosonic collective fields are now expressed in terms of unitary operators belonging to the fundamental representation of $S U(2 K)$, with $K \rightarrow \infty$. Similarly, the operator $\hat{\rho}_{0}$ of section I is given in the present context by

$$
\hat{\rho_{0}}=\sum_{n=0}^{N-1}|n\rangle\langle n| \Omega
$$

where $\Omega$ is a $2 \times 2$ Hermitean matrix incorporating the information about the spin of the many body ground state. Since the ground state of the system is ferromagnetic, $\Omega=P_{+} \equiv \frac{1}{2}\left(I+\sigma_{3}\right)$. $P_{+}$is the projector onto the spin "up" state.

We can express the operator $\hat{\rho}_{0}$ in the coherent state basis.

$$
\rho_{0}\left(|z|^{2}\right) \equiv e^{-|z|^{2}}\left\langle\bar{z}\left|\hat{\rho}_{0}\left(\hat{a}, \hat{a}^{\dagger}\right)\right| z\right\rangle
$$

For a large number of particles $(N \rightarrow \infty)[5]$,

$$
\rho_{0}\left(|z|^{2}\right)=\theta\left(N-|z|^{2}\right) P_{+}
$$

This immediately tells us that in order to convert this into a statement about the spatial extent of the system, we should require that $|z|^{2}=\frac{N}{R^{2}} r^{2}$, whence the theta function would look like $\theta\left(N-\frac{N}{R^{2}} r^{2}\right)=\theta\left(R^{2}-r^{2}\right)$. This in turn tells us that $z \sim N$.

Let us now, as a preview of the techniques to be presented in reasonable detail in the following section, look at a simple calculation. We shall look at $\operatorname{tr} \hat{\rho_{0}} \ddagger \hat{A} \ddagger \ddagger \hat{B} \ddagger$, where $A, B$ are arbitrary functions of $\hat{a}, \hat{a^{\dagger}}$. By introducing the coherent states, it is given by

$$
\operatorname{tr} \int d^{2} z e^{-|z|^{2}} \hat{\rho_{0}}|z\rangle A * B\langle\bar{z}|=\operatorname{tr} \int d^{2} z \rho_{0}\left(|z|^{2}\right) A * B
$$

In the last expression the trace is over the spin indices alone. 


\section{Effective Lagrangian for the Collective Excitations of the Quantum Hall Ferromagnet}

In this section, we shall present a somewhat detailed derivation of the bosonic effective Lagrangian given in (1.7), for the case of the quantum Hall ferromagnet. In the sequel, we shall take all the operators to have been projected onto the L.L.L..

Since the operator $\hat{U}$ is unitary, we can write it as $\hat{U} \equiv e^{i \hat{A}}$, where $\hat{A}$ is a Hermitean operator. Let us also define the function $g(z, \bar{z}) \equiv e^{i A(z, \bar{z})}$. As we have noted in the previous section, $z \sim \sqrt{N}$. Thus a derivative with respect to $z$, acting on $U(z, \bar{z})$ will carry with it a factor of $N^{-\frac{1}{2}}$. For a large value of $N$, we thus have a natural parameter to expand $U$ in. In fact [5],

$$
U(z, \bar{z})=g(z, \bar{z})-g_{, \bar{z}, z}+O\left(\frac{1}{N^{2}}\right)
$$

where $g_{, \bar{z}, z} \equiv i g \int_{0}^{1} d \alpha e^{-i \alpha A} \partial_{\bar{z}} A \partial_{z} e^{i \alpha A}$.

The effective Lagrangian may be represented as

$$
L_{e f f}=\mathcal{A}+\mathcal{B}+\mathcal{C}+\mathcal{D}
$$

where

$$
\begin{gathered}
\mathcal{A} \equiv \operatorname{tr} \hat{\rho} \hat{U}^{\dagger} i \partial_{t} \hat{U} \\
\mathcal{B} \equiv-\operatorname{tr} \hat{\rho} \hat{U}^{\dagger} \hat{v}_{c} \hat{U} \\
\mathcal{C} \equiv \frac{1}{2} \int d \vec{k} V(|\vec{k}|) \operatorname{tr}\left(\hat{\rho} \hat{U}^{\dagger} e^{-i \hat{\chi}} \hat{U} \hat{\rho} \hat{U}^{\dagger} e^{i \hat{\chi}} \hat{U}\right) \\
\mathcal{D} \equiv-\frac{1}{2} \int d \vec{k} V(|\vec{k}|) \operatorname{tr}\left(\hat{\rho} \hat{U}^{\dagger} e^{-i \hat{\chi}} \hat{U}\right) \operatorname{tr}\left(\hat{\rho} \hat{U}^{\dagger} e^{i \hat{\chi}} \hat{U}\right)
\end{gathered}
$$

where $V(|\vec{k}|)$ is the Fourier transform of the Coulomb potential and $e^{i \hat{\chi}} \equiv e^{i \bar{k} a} e^{i k a^{\dagger}}$, with $k \equiv \frac{1}{\sqrt{2 B}}\left(k_{x}+i k_{y}\right)$. The third and the fourth terms in the effective Lagrangian represent the contributions of the exchange and the direct parts respectively of the Coulomb term.

Using the coherent state representation and the large $N$ limit of the density, we can show that $[8]$

$$
\begin{aligned}
\mathcal{A} & =\int d^{2} z \theta\left(N-|z|^{2}\right) \operatorname{tr} P_{+} g^{\dagger} i \partial_{t} g \\
& +\frac{i}{2} \int d^{2} z \delta\left(N-|z|^{2}\right) \operatorname{tr} P_{+}\left(g^{\dagger}\left(\bar{z} \partial_{\bar{z}}-z \partial_{z}\right) g g^{\dagger} \partial_{t} g+\left[g^{\dagger} z \partial_{z} g, g^{\dagger} \partial_{t} g\right]\right) .
\end{aligned}
$$


The first term is obviously a bulk term and the second, an edge term.

Similarly,

$$
\begin{gathered}
\mathcal{B}=\int d^{2} z \theta\left(N-|z|^{2}\right)\left[-v_{c}+\operatorname{tr} P_{+}\left(\partial_{z} v_{c} g^{\dagger} \partial_{\bar{z}} g-\partial_{\bar{z}} v_{c} g^{\dagger} \partial_{z} g\right)\right] \\
+\int d^{2} z \delta\left(N-|z|^{2}\right) v_{c} \operatorname{tr} P_{+} g^{\dagger} \partial_{\bar{z}} g g^{\dagger} \partial_{z} g \\
\mathcal{C}=-\frac{1}{2 l} \sqrt{\frac{\pi}{2}} \int d^{2} z \theta\left(N-|z|^{2}\right)\left[\operatorname{tr} P_{+} g^{\dagger} \partial_{\bar{z}} g g^{\dagger} \partial_{z} g-\operatorname{tr} P_{+} g^{\dagger} \partial_{\bar{z}} g \operatorname{tr} P_{+} g^{\dagger} \partial_{z} g\right] \\
+\frac{1}{4 l} \sqrt{\frac{\pi}{2}} \int d^{2} z \delta\left(N-|z|^{2}\right) \operatorname{tr} P_{+} g^{\dagger}\left(\bar{z} \partial_{\bar{z}}-z \partial_{z}\right) g . \\
\mathcal{D}=\frac{1}{2} \int d^{2} z_{1} d^{2} z_{2} V\left(\left|z_{1}-z_{2}\right|\right) \times \\
\left(-\theta\left(N-\left|z_{1}\right|^{2}\right) \operatorname{tr} P_{+}\left[g^{\dagger} \partial_{\bar{z}_{1}} g, g^{\dagger} \partial_{z_{1}} g\right]+\delta\left(N-\left|z_{1}\right|^{2}\right) \operatorname{tr} P_{+} g^{\dagger}\left(\bar{z}_{1} \partial_{\bar{z}_{1}}-z_{1} \partial_{z_{1}}\right) g\right) \\
\quad\left(-\theta\left(N-\left|z_{2}\right|^{2}\right) \operatorname{tr} P_{+}\left[g^{\dagger} \partial_{\bar{z}_{2}} g, g^{\dagger} \partial_{z_{2}} g\right]+\delta\left(N-\left|z_{2}\right|^{2}\right) \operatorname{tr} P_{+} g^{\dagger}\left(\bar{z}_{2} \partial_{\bar{z}_{2}}-z_{2} \partial_{z_{2}}\right) g\right) .
\end{gathered}
$$

A uniform background density of $\frac{B}{2 \pi}$ has been subtracted off from the direct contribution. The deviation, in the excited state from the uniform density of the ground state is given by

$$
\delta \rho(\vec{x}) \equiv\left(-\theta\left(N-|z|^{2}\right) \operatorname{tr} P_{+}\left[g^{\dagger} \partial_{\bar{z}} g, g^{\dagger} \partial_{z} g\right]+\delta\left(N-|z|^{2}\right) \operatorname{tr} P_{+} g^{\dagger}\left(\bar{z} \partial_{\bar{z}}-z \partial_{z}\right) g\right)
$$

From the above, it is clear that the contributions to the effective Lagrangian split naturally into bulk and edge contributions.

In obtaining (3.19) and (3.10), we have used the following relations:

$$
\left\langle\bar{z}_{2}|\hat{f}| z_{1}\right\rangle=e^{\overline{z_{2}} z_{1}} \int d^{2} w d^{2} z e^{-|z|^{2}} e^{\bar{w}\left(z-z_{1}\right)-w\left(\bar{z}-\overline{z_{2}}\right)}\langle\bar{z}|\hat{f}| z\rangle
$$

and

$$
\int d^{2} \eta f(\eta, \bar{\eta}) \int d^{2} w e^{\bar{w}(\eta-z)-w(\bar{\eta}-\bar{z})}=f(z, \bar{z})
$$

\section{Scaling of the terms in the bosonic effective Lagrangian with $N$}


In the preceding section, we have obtained the leading contributions to the bosonic effective Lagrangian that emerge from the underlying microscopic fermionic action. We have considered the number of particles in the system, $N \gg 1$ and have developed a systematic derivative expansion scheme, with $1 / \sqrt{N}$ as the small parameter, to identify the leading contributions. In this section, we shall study how the various terms scale with $N$ and rewrite the various terms as integrals over real spatial coordinates. In equation (3.7), we note that in order to write the theta function in terms of spatial coordinates, we have to take $z=\frac{\sqrt{N}}{R} \eta$, where $R$ is the radius of the droplet given by $R=\sqrt{\frac{2 N}{B}}$ and $\eta \equiv x+i y$. Then we get $\theta\left(N-|z|^{2}\right)=\theta\left(R^{2}-r^{2}\right)$. This just means that the bulk contribution has support inside of the droplet. The measure $d^{2} z$ then becomes $\frac{B}{2 \pi} d x d y$. Furthermore, we note that since $\frac{B}{2 \pi} \pi R^{2}=N, N=\frac{B R^{2}}{2}$. Thus, we have, from (3.7),

$$
\mathcal{A}_{\text {bulk }}=i \frac{B}{2 \pi} \int d \vec{x} \theta\left(R^{2}-r^{2}\right) \operatorname{tr} P_{+} g^{\dagger} \partial_{t} g
$$

This term is proportional to the area of the droplet and hence is proportional to $N$. Again, from (3.7), we have,

$$
\mathcal{A}_{\text {edge }}=\frac{1}{8 \pi} \int_{0}^{2 \pi} d \theta \operatorname{tr} P_{+}\left(\left\{g^{\dagger} i \partial_{\theta} g, g^{\dagger} i \partial_{t} g\right\}+\left[g^{\dagger} r \partial_{r} g, g^{\dagger} i \partial_{t} g\right]\right)_{r=R}
$$

This term is of $O(1)$ in $N$ as expected as it is a boundary term and as such should be independent of the number of particles in the droplet.

The contributions due to the confining potential are given in (3.8). We have argued previously that for a proper thermodynamic limit to exist, that is for the energy of the droplet to scale as $N$, the confining potential should be of order one (in $N$ ) in the bulk. Alternatively, $\partial_{z} v \sim 1 / \sqrt{N}$ in the bulk. Thus, we see that the second term in $\mathcal{B}$ is an order one contribution to the bulk effective Lagrangian. Thus it is a subleading contribution (compared to $\mathrm{O}(N)$ contributions) and may be dropped. Again,

$$
\mathcal{B}_{\text {edge }}=-\frac{\omega}{8 \pi} \int_{0}^{2 \pi} d \theta \operatorname{tr} P_{+}\left(-\left(g^{\dagger} r \partial_{r} g\right)^{2}+\left[g^{\dagger} r \partial_{r} g, g^{\dagger} i \partial_{\theta} g\right]+\left(g^{\dagger} i \partial_{\theta} g\right)^{2}\right)_{r=R}
$$

where $\omega \equiv \frac{v(r=R)}{N}$, and is of order one in $N$.

Let us now turn our attention to the contributions of the Coulomb term.

$$
\mathcal{C}_{b u l k}=-\frac{1}{2 l} \frac{1}{\sqrt{2 \pi}} \int d \vec{x} \theta\left(R^{2}-r^{2}\right)\left[\operatorname{tr} P_{+} g^{\dagger} \partial_{\bar{\eta}} g g^{\dagger} \partial_{\eta} g-\operatorname{tr} P_{+} g^{\dagger} \partial_{\bar{\eta}} g \operatorname{tr} P_{+} g^{\dagger} \partial_{\eta} g\right]
$$


Similarly, using $\bar{\eta} \partial_{\bar{\eta}}-\eta \partial_{\eta}=i \partial_{\theta}$, where $\theta$ is the plane polar angle, we get

$$
\mathcal{C}_{\text {edge }}=\left.\frac{1}{8 l} \frac{1}{\sqrt{2 \pi}} \int_{0}^{2 \pi} d \theta \operatorname{tr} P_{+} g^{\dagger} i \partial_{\theta} g\right|_{r=R}
$$

The contribution in (4.4) is proportional to the area of the droplet and is thus of order $N$, whilst that in (4.5) is of order one in $N$.

Similar arguments can be provided for the contributions from the direct part of the Coulomb interaction.

The the leading contributions to the effective Lagrangian, governing the collective excitations in the bulk, are given by

$$
\begin{aligned}
& L_{\text {eff }}^{(\text {bulk })}=\int_{\mathcal{D}} d \vec{x}\left[\frac{B}{2 \pi} \operatorname{tr} P_{+} g^{\dagger} i \partial_{t} g-\frac{1}{2 l} \frac{1}{\sqrt{2 \pi}}\left(\operatorname{tr} P_{+} g^{\dagger} \partial_{\bar{\eta}} g g^{\dagger} \partial_{\eta} g-\operatorname{tr} P_{+} g^{\dagger} \partial_{\bar{\eta}} g \operatorname{tr} P_{+} g^{\dagger} \partial_{\eta} g\right)\right] \\
& +\frac{1}{2 \pi^{2}} \int_{\mathcal{D}} d \vec{x}_{1} \int_{\mathcal{D}} d \vec{x}_{2} V\left(\left|\vec{x}_{1}-\vec{x}_{2}\right|\right)\left(\operatorname{tr} P_{+}\left[g^{\dagger} \partial_{\overline{\eta_{1}}} g, g^{\dagger} \partial_{\eta_{1}} g\right]\right)\left(\operatorname{tr} P_{+}\left[g^{\dagger} \partial_{\overline{\eta_{2}}} g, g^{\dagger} \partial_{\eta_{2}} g\right]\right)
\end{aligned}
$$

The subscript $\mathcal{D}$ indicates that the integral is over all $r<R$.

Similarly, we obtain the effective Lagrangian governing the collective dynamics at the edge of the droplet

$$
\begin{aligned}
L_{e f f}^{(e d g e)}= & \int_{0}^{2 \pi} d \theta\left[\frac{1}{8 \pi} \operatorname{tr} P_{+}\left(\left\{g^{\dagger} i \partial_{\theta} g, g^{\dagger} i \partial_{t} g\right\}+\left[g^{\dagger} r \partial_{r} g, g^{\dagger} i \partial_{t} g\right]\right)_{r=R}\right. \\
& -\frac{\omega}{8 \pi} \operatorname{tr} P_{+}\left(-\left(g^{\dagger} r \partial_{r} g\right)^{2}+\left[g^{\dagger} r \partial_{r} g, g^{\dagger} i \partial_{\theta} g\right]+\left(g^{\dagger} i \partial_{\theta} g\right)^{2}\right)_{r=R} \\
& \left.-\left.\frac{1}{2 l} \frac{1}{\sqrt{2 \pi}} \operatorname{tr} P_{+} g^{\dagger} i \partial_{\theta} g\right|_{r=R}\right] \\
& -\left.\frac{1}{8 \pi} \int_{0}^{2 \pi} d \theta_{1} d \theta_{2} V\left(\left|\vec{x}_{1}-\vec{x}_{2}\right|\right)\left(\operatorname{tr} P_{+} g^{\dagger} i \partial_{\theta_{1}} g\right)\left(\operatorname{tr} P_{+} g^{\dagger} i \partial_{\theta_{2}} g\right)\right|_{r_{1}, r_{2}=R}
\end{aligned}
$$

At this point, we note that if $\hat{\rho_{0}}=\sum_{n=0}^{\infty}|n\rangle\langle n| P_{+}$, all the available s.p. states in the L.L.L. would have been filled and the effective Lagrangian would have been entirely a bulk effective Lagrangian (as the droplet would in this case fill the entire plane). In fact

$$
\rho_{0}\left(|z|^{2}\right) \equiv e^{-|z|^{2}}\left\langle\bar{z}\left|\hat{\rho_{0}}\right| z\right\rangle=P_{+}
$$

Thus, the effective Lagrangian would be given by (4.6) with the support of the integral over $x, y$ extending over the entire droplet. Namely,

$$
\begin{aligned}
& L_{e f f}^{(\text {bulk })}=\int d \vec{x}\left[\frac{B}{2 \pi} \operatorname{tr} P_{+} g^{\dagger} i \partial_{t} g-\frac{1}{2 l} \frac{1}{\sqrt{2 \pi}}\left(\operatorname{tr} P_{+} g^{\dagger} \partial_{\bar{\eta}} g g^{\dagger} \partial_{\eta} g-\operatorname{tr} P_{+} g^{\dagger} \partial_{\bar{\eta}} g \operatorname{tr} P_{+} g^{\dagger} \partial_{\eta} g\right)\right] \\
& +\frac{1}{2 \pi^{2}} \int_{\mathcal{D}} d \vec{x}_{1} \int_{\mathcal{D}} d \vec{x}_{2} v_{c}\left(\left|\vec{x}_{1}-\vec{x}_{2}\right|\right)\left(\operatorname{tr} P_{+}\left[g^{\dagger} \partial_{\overline{\eta_{1}}} g, g^{\dagger} \partial_{\eta_{1}} g\right]\right)\left(\operatorname{tr} P_{+}\left[g^{\dagger} \partial_{\overline{\eta_{2}}} g, g^{\dagger} \partial_{\eta_{2}} g\right]\right)
\end{aligned}
$$


This is the effective Lagrangian that has been discussed extensively in the literature, in the context of ferromagnetic magnons in the Hall ferromagnet [1],[7].

\section{Simplifying the effective Lagrangian}

In this section, we shall look closely at the various contributions to the bulk and the edge effective Lagrangians and comment on their relative importance. We shall look separately at the the bulk and the edge contributions.

In terms of the familiar Euler angles, we can parametrise $g$ as

$$
g \equiv e^{-i \frac{\phi}{2} \sigma_{3}} e^{-i \frac{\theta}{2} \sigma_{2}} e^{-i \frac{\chi}{2} \sigma_{3}}
$$

with

$$
g \sigma_{3} g^{\dagger} \equiv \hat{m} \cdot \vec{\sigma}, \hat{m}^{2}=1
$$

where

$$
\hat{m}=(\sin \theta \cos \phi, \sin \theta \sin \phi, \cos \theta)
$$

Again, we may parametrise $g$ in a more conventional manner as

$$
\begin{aligned}
g & \equiv e^{i \vec{A} \cdot \vec{\sigma}} \\
g^{\dagger} i \partial_{\mu} g & =-\vec{\sigma} \cdot\left(\partial_{\mu} \vec{A}+\vec{A} \times \partial_{\mu} \vec{A}\right) .
\end{aligned}
$$

where we have retained upto the quadratic in $\vec{A}$. Furthermore, we shall assume the following ansatz for $\vec{A}$,

$$
\vec{A}=\frac{1}{2}\left(m_{2},-m_{1}, 0\right)
$$

and consider $m_{3} \simeq 1$, with $\left|\vec{m}_{T}\right| \ll 1$, where $\vec{m}_{T} \equiv\left(m_{1}, m_{2}, 0\right)$.

Then the various contributions to the bulk effective Lagrangian may be written as:

$$
\begin{aligned}
\mathcal{A}_{\text {bulk }} & =-\frac{1}{2} \frac{B}{2 \pi} \int_{D} d \vec{x}(1-\cos \theta) \partial_{t} \phi \\
& =\frac{1}{2} \frac{B}{2 \pi} \int_{D} d \vec{x} \int_{0}^{1} d \lambda \hat{m}_{\lambda}(\vec{x}, t) \cdot\left[\partial_{t} \hat{m}_{\lambda}(\vec{x}, t) \times \partial_{\lambda} \hat{m}_{\lambda}(\vec{x}, t)\right]
\end{aligned}
$$


where $\hat{m}_{\lambda} \equiv \hat{m}(\lambda \theta, \phi)$. We require $\hat{m}_{\lambda=0}=\hat{e}_{3}$ and $\hat{m}_{\lambda=1}=\hat{m}$. Explicitly, we may choose

$$
\hat{m}_{\lambda}(\theta, \phi)=(\sin \lambda \theta \sin \phi, \sin \lambda \theta \cos \phi, \cos \lambda \theta) .
$$

The second line in (5.5) is the well-known Wess-Zumino term and is proportional to the area of the droplet. The suffix $D$ indicates that the integral has support over the entire area of the droplet.

As we have argued before, we may drop $\mathcal{B}_{\text {bulk }}$.

$$
\mathcal{C}_{\text {bulk }}=-\frac{1}{32 l \sqrt{2 \pi}} \int_{D} d \vec{x}\left(\partial_{\alpha} \hat{m}\right)^{2}-\frac{1}{4 l} \sqrt{\frac{\pi}{2}} \int_{D} d \vec{x} \rho_{p}(\vec{x}, t)
$$

where $\rho_{p} \equiv-\frac{1}{8 \pi} \epsilon_{\alpha \beta} \hat{m} \cdot\left(\partial_{\alpha} \hat{m} \times \partial_{\beta} \hat{m}\right)$ is the well known Pontryagin index density. The integral of $\rho_{p}$ over all space gives an integer.

$$
\mathcal{D}_{b u l k}=-\frac{1}{2} \int_{D} d \vec{x}_{1} d \vec{x}_{2} \rho_{p}\left(\vec{x}_{1}, t\right) V\left(\left|\vec{x}_{1}-\vec{x}_{2}\right|\right) \rho_{p}\left(\vec{x}_{2}, t\right)
$$

Thus, the bulk effective Lagrangian is given by:

$$
\begin{aligned}
L_{e f f}^{(b u l k)} & =\int_{D} d \vec{x}\left[\frac{B}{4 \pi} \int_{0}^{1} d \lambda \hat{m}_{\lambda} \cdot\left[\partial_{t} \hat{m}_{\lambda} \times \partial_{\lambda} \hat{m}_{\lambda}\right]-\frac{1}{32 l \sqrt{2 \pi}}\left(\partial_{\alpha} \hat{m}\right)^{2}-\frac{1}{4 l} \sqrt{\frac{\pi}{2}} \rho_{p}\right] \\
& -\frac{1}{2} \int_{D} d \vec{x}_{1} d \vec{x}_{2} \rho_{p}\left(\vec{x}_{1}\right) V\left(\left|\vec{x}_{1}-\vec{x}_{2}\right|\right) \rho_{p}\left(\vec{x}_{2}\right) .
\end{aligned}
$$

This Lagrangian has been obtained in a variety of ways in the literature. It governs the dynamics of the ferromagnetic magnons [1],[7].

Let us now look at the effective Lagrangian governing the edge excitations. To simplify matters, we shall focus on those excitations that satisfy the condition $\left.\partial_{r} \hat{m}(r, \theta, t)\right|_{r=R}=0$.

Then,

$$
\mathcal{A}_{\text {edge }} \simeq \frac{1}{16 \pi} \int_{0}^{2 \pi} d \theta\left[\partial_{t} \hat{m} \cdot \partial_{\theta} \hat{m}\right]_{r=R}
$$

Further,

$$
\mathcal{B}_{\text {edge }} \simeq-\frac{\omega}{32 \pi} \int_{0}^{2 \pi} d \theta\left[\left(\partial_{\theta} \hat{m}\right)^{2}\right]_{r=R}
$$

where $\omega \equiv \frac{v_{c}(r=R)}{N}$.

Interestingly enough, to the leading order, the contributions of the Coulomb interaction to the Lagrangian for the edge, are zero. 
Thus,

$$
L_{e f f}^{(e d g e)}=\frac{1}{16 \pi} \int_{0}^{2 \pi} d \theta\left[\partial_{t} \hat{m} \cdot \partial_{\theta} \hat{m}-\frac{\omega}{2}\left(\partial_{\theta} \hat{m}\right)^{2}\right]_{r=R}
$$

These edge excitations are obviously chiral in nature.

We see that even though the bulk modes (ferromagnetic magnons) and the edge modes (chiral excitations) are both gapless, they owe their dynamics to completely different sources. For the bulk modes, which are the Goldstone modes corresponding to a spontaneous breaking of the global spin symmetry $S U(2) \rightarrow U(1)$, the exchange part of the Coulomb interaction is not only crucial to their dynamics, but is truly their raison d'etre.

On the other hand, the chiral edge excitations are somewhat generic to confined Hall fluids. Let us consider a specific instance. We shall consider a conventional $\nu=1$ Hall droplet where the spin degrees of freedom are completely frozen by the Zeeman term. In this case, $\rho\left(|z|^{2}\right)=\theta\left(N-|z|^{2}\right)$ and $g$ is simply a $U(1)$ phase. The edge contribution to to the effective Lagrangian can be easily shown to be

$$
L_{\text {eff }}^{(e d g e)}=\frac{1}{2 \pi} \int_{0}^{2 \pi} d \theta\left[\partial_{\theta} \Phi \partial_{t} \Phi-\frac{\omega}{2}\left(\partial_{\theta} \Phi\right)^{2}\right]
$$

This is the familiar Lagrangian obtained in the literature [9] for a case where the Coulomb interaction is known to be unimportant. The corresponding bulk contribution trivially vanishes. This should convince us that the edge excitations are extremely ubiquitous and as such do not owe their existence to the Coulomb interaction.

\section{Conclusions}

In this article, we have utilised the bosonisation technique introduced in [5] to discuss the collective excitations of a finite-sized Hall sample where $g \rightarrow 0$. For a large sample, (Area $\gg \frac{1}{B}$ ), we know that these excitations are [1],[7] ferromagnetic magnons whose dynamics is governed by the exchange part of the Coulomb interaction between the electrons. For a finite sample, we also need to consider the dynamics of the edge.

We have shown that the effective Lagrangian for the collective excitations splits naturally into two pieces, one having support in the bulk of the droplet and the other at the edge.

The bulk effective Lagrangian coincides with that computed for an infinite sample [1],[7]. The edge excitations, which are chiral in nature, are to the leading order, unaffected by the Coulomb 
interaction between the electrons. The mere fact that the electrons are confined to a droplet suffices to produce chiral edge excitations. 


\section{Acknowledgements}

This work was done collaboratively with Prof. B. Sakita. I hereby acknowledge his unstinting

help. I also thank Prof. D. Karabali for useful discussions and the organisers of the SCES-y2k conference for inviting me to give a talk.

\section{References}

[1] S.L. Sondhi et. al. Phys. Rev. B 47, 16419, (1993); K. Moon et. al. Phys. Rev. B 51, 5138, (1995)

[2] X.G. Wen, Phys. Rev. B 41, 12838, (1990); M. Stone, Ann. Phys. (N.Y.), 207, 38, (1991); Phys. Rev. B 53, 16573, (1996)

[3] R. Ray, B. Sakita, Ann. Phys. (N.Y.), 230, 131, (1994)

[4] M.V. Milovanovic, Phys. Rev. B 57, 9920, (1998); A. Karlhede et. al., Phys. Rev. B 60, 15948, (1999); Phys. Rev. Lett. 77, 2061, (1996); T.H. Hansson, S. Viefers, Phys. Rev. B 61, 7553, (2000)

[5] B. Sakita, Phys. Lett. B 387, 118, (1996)

[6] J.P. Blaizot, H. Orland, Phys. Rev. C 24, 1740, (1981); H. Kuratsuji, T. Suzuki, Prog. Theor. Phys. Suppl. 75 and 76, 209, (1983); A. Dhar et. al., Mod. Phys. Lett. A 8, 3557, (1993)

[7] R. Ray, Phys. Rev. B60, 14154, (2000)

[8] R. Ray, B. Sakita, (in preparation)

[9] S. Iso et. al., Nucl. Phys. B 388, 700, (1992); Phys. Lett. B 296, 143, (1992) 